\title{
SYMMETRIZATION WITH RESPECT TO A MEASURE
}

\author{
FRIEDMAR SCHULZ AND VIRGINIA VERA DE SERIO
}

\begin{abstract}
In this paper we study the spherical symmetric rearrangement $u^{*}$ of a nonnegative measurable function $u$ on $\mathbb{R}^{n}$ with respect to a measure given by a nonhomogeneous density distribution $p$. Conditions on $u$ are given which guarantee that $u^{*}$ is continuous, of bounded variation, or absolutely continuous on lines, i.e., Sobolev regular. The energy inequality is proven in $n=2$ dimensions by employing a Carleman type isoperimetric inequality if $\log p$ is subharmonic. The energy equality is settled via a reduction to the case of a homogeneous mass density.
\end{abstract}

\section{INTRODUCTION}

A brief description of the spherical symmetric rearrangement $u^{*}$ with respect to a measure $\mathscr{M}^{n}$ of a nonnegative measurable function $u$ with compact support in $\mathbb{R}^{n}$ is as follows: $u^{*}$ is a radially symmetric function, $u^{*}(x)=\tilde{u}(|x|)$, where $\tilde{u}$ is a monotone nonincreasing function which is continuous from the right, such that the distribution functions of $u$ and $u^{*}$ are equal:

$$
\mu(t)=\mathscr{L}^{n}\left(u^{*-1}(t, \infty)\right)=\mathscr{M}^{n}\left(u^{-1}(t, \infty)\right)
$$

for all $t \in \mathbb{R}$.

The spherical symmetric rearrangement $u^{*}$ with respect to the Lebesgue measure $\mathscr{L}^{n}$ is the classical symmetric rearrangement by Schwarz. Under minimal regularity assumptions regarding $u$, it follows readily from the BrunnMinkowski inequality that $u^{*}$ is Lipschitz continuous. An important property is that the energy decreases:

$$
\int_{\mathbb{R}^{n}}\left|D u^{*}\right|^{2} d \mathscr{L}^{n} \leq \int_{\mathbb{R}^{n}}|D u|^{2} d \mathscr{L}^{n}
$$

Recently Brothers and Ziemer [BZ] settled the discussion of the energy equality: If $u$ is a Sobolev function of class $W^{1,2}\left(\mathbb{R}^{n}\right)$, then the energy equality takes place if and only if $u$ is almost everywhere equal to a translate of $u^{*}$. The crucial assumption here is that $\mathscr{L}^{n}\left(u^{*-1}(0, M) \cap\left\{x: D u^{*}(x)=0\right\}\right)=0$ $\left(M=\operatorname{ess} \sup u^{*}=\right.$ ess $\left.\sup u\right)$, which is equivalent to the absolute continuity of the distribution function $\mu$ of $u^{*}$ or $u$. Earlier results were obtained by

Received by the editors July 17, 1990 and, in revised form, January 23, 1991.

1980 Mathematics Subject Classification (1985 Revision). Primary 26D15; Secondary 26B15, 26B30, 28A75, 30C20, 49F10, 49G05, 51M25.

Key words and phrases. Schwarz symmetrization, spherical symmetric rearrangement, isoperimetric inequality, energy inequality, Faber-Krahn inequality, nonhomogeneous mass density, coarea formula, Sobolev function, function of bounded variation. 
Kawohl (see $[\mathrm{K}]$ ), who assumed the analyticity of $u$, by Friedman and McLeod [FM] (here $u \in C^{n}$ ), whose proof can be corrected under the stronger assumption that $\mathscr{L}^{n}\left(u^{-1}(0, M) \cap\{x: D u(x)=0\}\right)=0$, and also by Schulz [S], where the energy equality was settled in the case that $u$ is the first eigenfunction of the Dirichlet problem for the Laplacian in a Dirichlet domain (strict inequality in Faber-Krahn's inequality).

However, if one symmetrizes $u$ with respect to a nonhomogeneous measure $\mathscr{M}^{n}$ given by a density distribution $p$, then $u^{*}$ may not be Lipschitz continuous, and approximation arguments to show Sobolev regularity therefore break down. The energy inequality, which rests on an isoperimetric inequality, may only be proven under suitable assumptions regarding $p$.

In $\S 1$ we shall derive some basic regularity properties of $u^{*}$ under minimal assumptions regarding $u$, and without requiring anything about $p$ except that it is a positive, measurable, and locally integrable function. The Sobolev regularity $u^{*} \in W^{1,1}$ is e.g. proven if $u \in W^{1,1} \cap L^{\infty}$, and $u^{*} \in W_{\text {loc }}^{1,1}\left(\mathbb{R}^{n} \backslash\{0\}\right)$ if $u \in W^{1,1}$.

In $\S 2$ we shall prove the energy inequality for Sobolev functions of class $W^{1,2}\left(\mathbb{R}^{2}\right)$ in the plane $(n=2)$ and settle the equality. Crucial to our investigation is the conformal invariance of the Dirichlet integral. First a weighted isoperimetric inequality, which is due to Carleman [C], is generalized to sets of finite perimeter by following the scheme of proving the equivalence of Sobolev and isoperimetric inequalities. The assumption here is that the logarithm of the density distribution $p$ is subharmonic. The energy equality is settled for Sobolev functions for which $u^{-1}(0, \infty)$ is bounded by finitely many disjoint Jordan curves if $\mathscr{L}^{n}\left(u^{*-1}(0, M) \cap\left\{x: D u^{*}(x)=0\right\}\right)=0$. Equality then holds if and only if $u$ is almost everywhere equal to $u^{*} \circ F$, where $F$ is a one-to-one conformal map onto a disk $D^{*}$ such that $p=\left|F^{\prime}\right|^{2}$.

We shall generally follow the Diplomarbeit of Schulz [S] and the Dissertation of Vera de Serio [VS] which freely utilize the expositions about Schwarz symmetrization by Brothers and Ziemer [BZ], Hildén [H], Sperner [SP], and Talenti [T]:

Section 1 depends largely on [BZ]; we follow [BZ] in particular to show the absolute continuity of $\tilde{u}(|x|)=u^{*}(x)$. However, we try to achieve an exposition which is as elementary as possible: In contrast to [BZ] we use only fairly wellknown facts about functions of bounded variation and sets of finite perimeter instead of geometric measure theory results on currents of dimension $n$ in $\mathbb{R}^{n}$ and a general GMT isoperimetric inequality. The expositions of Evans-Gariepy [EG] and Ziemer [Z] about BV-functions were particularly useful. Some facts which are not readily available in the literature are given; the proof of the Riesz representation theorem is crucial.

Section 2 builds on Schulz [S], which in turn rests on Sperner [SP]. The Dissertation of Vera de Serio [VS] improves on this by invoking classical complex variable methods: In the discussion of the energy equality, a reduction to the case of a homogeneous mass distribution is achieved by mapping level sets of $u$ conformally onto discs and proving a compactness theorem. In this article this is achieved by one conformal map by virtue of the lower semicontinuity of the variation measure and the isoperimetric inequality (see Lemma 2.4.2, which rests on Lemma 2.3.4). The results here are more general due to a measure- 
theoretic approach. Although only measure-theoretic properties about sets of finite perimeter are used in this article, ultimately, through the reduction to the homogeneous case and the citation of [BZ], the general GMT results mentioned above do in fact enter the final result about minimal rearrangements.

We are motivated by the following application to vibrating nonhomogeneous membranes: This article provides a rigorous proof of Nehari's generalization of the Faber-Krahn inequality [N], and the equality is settled as well, i.e., the fundamental frequency of a nonhomogeneous membrane with total mass $m$ is larger than or equal to the fundamental frequency of the circular homogeneous membrane whose area equals $m$, if the logarithm of the mass density is subharmonic. A final remark is, that in a differential geometric setting, the assumption about the subharmonicity of the density distribution corresponds to the nonpositivity of the Gauss curvature of the surface under consideration.

\section{DEFINITION AND REgUlARITY PROPERTIES ON $\mathbb{R}^{n}$}

1.1. Definition of the spherical symmetric rearrangement $u^{*}$. Let $\mathscr{L}^{n}$ denote the Lebesgue measure on $\mathbb{R}^{n}$, and consider the absolutely continuous measure $\mathscr{M}^{n}$ given by

$$
\mathscr{M}^{n}(A)=\int_{A} p d \mathscr{L}^{n}
$$

for any Lebesgue measurable set $A$ in $\mathbb{R}^{n}$. Here the density $p: \mathbb{R}^{n} \rightarrow \mathbb{R}^{+}, \mathbb{R}^{+}=$ $\{t>0\}$, is a positive, measurable, and locally integrable function. Throughout this article $u: \mathbb{R}^{n} \rightarrow \mathbb{R}_{0}^{+}, \mathbb{R}_{0}^{+}=\{t \geq 0\}$, is a nonnegative measurable function with compact support

$$
\operatorname{supp}(u)=\bar{E}_{0}, \quad E_{0}=u^{-1}(0, \infty) .
$$

The distribution function $\mu$ of $u$ with respect to the measure $\mathscr{M}^{n}$ is given by $\mu(t)=\mathscr{M}^{n}\left(E_{t}\right)$ for all $t \in \mathbb{R}$, where $E_{t}$ is the level set, $E_{t}=\mathcal{u}^{-1}(t, \infty) . \mu$ is a monotone nonincreasing function which is continuous from the right. Indeed, $\mu$ is discontinuous at every $t$ for which $\mathscr{L}^{n}\left(u^{-1}\{t\}\right)>0$, and $\mu\left(t^{-}\right)-\mu(t)=$ $\mathscr{M}^{n}\left(u^{-1}\{t\}\right)$.

1.1.1. Definition. The spherical symmetric rearrangement $u^{*}$ of $u$ with respect to the measure $\mathscr{M}^{n}$ is the function $u^{*}: \mathbb{R}^{n} \rightarrow \overline{\mathbb{R}}$ defined by

$$
u^{*}(x)=\sup \left\{t: \mu(t)>\alpha_{n}|x|^{n}\right\}
$$

whenever this set is nonempty, and $u^{*}(x)=0$ otherwise. Here $\alpha_{n}=\mathscr{L}^{n}\{x:|x|$ $<1\}$.

\subsection{Preliminary properties of $u^{*}$.}

1.2.1. Lemma. There exists a monotone nonincreasing function $\tilde{u}:[0, \infty) \rightarrow \overline{\mathbb{R}}$ such that $u^{*}(x)=\tilde{u}(|x|)$ for all $x \in \mathbb{R}^{n}$. $\tilde{u}$ is finite and continuous from the right on $(0, \infty)$, and $\tilde{u}(0)=\tilde{u}\left(0^{+}\right)=M=\operatorname{ess} \sup u$.

1.2.2. Corollary. The gradient of $u^{*}, D u^{*}$, exists almost everywhere on $\mathbb{R}^{n}$ and is a measurable function. Moreover $D u^{*}(x)=\tilde{u}^{\prime}(|x|) x /|x|$ for almost all $x$ in $\mathbb{R}^{n}$.

1.2.3. Lemma. Let $E_{t}$ and $E_{t}^{*}$ be the level sets of the functions $u$ and $u^{*}$ respectively. Then for all $t \geq 0, E_{t}^{*}$ is the open ball centered at the origin with 
$\mathscr{L}^{n}\left(E_{t}^{*}\right)=\mathscr{M}^{n}\left(E_{t}\right)$. In particular, $u^{*}$ has compact support $\bar{E}_{0}^{*}$ and takes values on $[0, M]$.

The equality of the distribution functions $\mu^{*}(t)=\mathscr{L}^{n}\left(E_{t}^{*}\right)$ and $\mu(t)=$ $\mathscr{M}^{n}\left(E_{t}\right)$ implies:

1.2.4. Corollary. For any nonnegative Borel measurable function $g: \mathbb{R} \rightarrow \mathbb{R}$, we have

$$
\int_{\mathbb{R}^{n}} g\left(u^{*}(x)\right) d \mathscr{L}^{n}=\int_{\mathbb{R}^{n}} g(u(x)) p(x) d \mathscr{L}^{n} .
$$

1.3. $u \in L^{\infty}\left(\mathbb{R}^{n}\right)$ implies $u^{*} \in \mathrm{BV}\left(\mathbb{R}^{n}\right)$. Recall that the total variation $\|D f\|$ of a locally integrable function $f$ on $\mathbb{R}^{n}$ is

$$
\|D f\|=\|D f\|\left(\mathbb{R}^{n}\right)=\sup \left\{\int_{\mathbb{R}^{n}} f \operatorname{div} g d \mathscr{L}^{n}: g \in C_{0}^{\infty}\left(\mathbb{R}^{n}, \mathbb{R}^{n}\right),|g| \leq 1\right\} .
$$

The function $f$ is of bounded variation, written $f \in \mathrm{BV}\left(\mathbb{R}^{n}\right)$, if $f \in L^{1}\left(\mathbb{R}^{n}\right)$ and $\|D f\|<\infty$. If the gradient of $f$ exists in the distributional sense, then

$$
\|D f\|=\int_{\mathbb{R}^{n}}|D f| d \mathscr{L}^{n}
$$

1.3.1. Theorem. If $u \in L^{\infty}\left(\mathbb{R}^{n}\right)$, then $u^{*} \in \mathrm{BV}\left(\mathbb{R}^{n}\right)$, and the total variation of $u^{*}$ is

$$
\left\|D u^{*}\right\|=-n \alpha_{n} \int_{0}^{\infty} r^{n-1} d \tilde{u}(r)
$$

Proof. Let $g \in C_{0}^{\infty}\left(\mathbb{R}^{n}, \mathbb{R}^{n}\right)$ with $|g| \leq 1$. Then

$$
\begin{aligned}
\int_{\mathbb{R}^{n}} u^{*} \operatorname{div} g d \mathscr{L}^{n} & =\int_{0}^{\infty} \tilde{u}(r) \int_{|x|=r} \operatorname{div} g d \mathscr{H}^{n-1} d r \\
& =-\int_{0}^{\infty} \int_{|x| \leq r} \operatorname{div} g d \mathscr{L}^{n} d \tilde{u}(r) \\
& =-\int_{0}^{\infty} \int_{|x|=r} g \cdot x /|x| d \mathscr{H}^{n-1} d \tilde{u}(r) .
\end{aligned}
$$

Here $\mathscr{H}^{n-1}$ denotes the $(n-1)$-dimensional Hausdorff measure. Therefore

$$
\left\|D u^{*}\right\| \leq-n \alpha_{n} \int_{0}^{\infty} r^{n-1} d \tilde{u}(r)
$$

Equality follows by approximating $x /|x|$ by test functions $g,|g| \leq 1$, as required.

1.4. $u \in C^{0}\left(\mathbb{R}^{n}\right)$ implies $u^{*} \in C^{0}\left(\mathbb{R}^{n}\right)$. For any $t \in \mathbb{R}$ let $\lambda(t)=\mathscr{M}^{n}\left(u^{-1}[t, \infty)\right)$. Then $\lambda$ is monotone nonincreasing, continuous from the left, $\mu(t)=\lambda\left(t^{+}\right)$, and $\lambda(t)=\mu\left(t^{-}\right)$.

1.4.1. Lemma. If $u \in C^{0}\left(\mathbb{R}^{n}\right)$, then $\lambda(s)<\mu(t)$ for all $t, s, 0 \leq t<M$, $t<s$. Moreover, $\mu$ and $\lambda$ are (strictly) decreasing functions on $[0, M]$.

For all $t \geq 0$ let $A_{t}=\left\{x: \mu(t)>\alpha_{n}|x|^{n}\right\}$ and $B_{t}=\left\{x: \lambda(t) \geq \alpha_{n}|x|^{n}\right\}$ if $0 \leq t \leq M$, and $B_{t}=\varnothing$ otherwise, and put $C_{t}=B_{t} \backslash A_{t}$. 
1.4.2. Lemma. For any $t \geq 0$,

$$
\begin{aligned}
& A_{t}=\bigcup_{s>t} B_{s}, \\
& B_{t}=\bigcup_{s \geq t} C_{s}, \\
& A_{t}=\bigcup_{s>t} C_{s} .
\end{aligned}
$$

Proof. Assume that $t<M$, and let $x \in B_{t}$, i.e., $\lambda(t) \geq \alpha_{n}|x|^{n}$. Put $s_{0}=$ $\sup \left\{s: \alpha_{n}|x|^{n} \leq \lambda(s)\right\}$. Then $s_{0} \geq t$ and $\alpha_{n}|x|^{n} \leq \lambda\left(s_{0}\right) \leq \lambda(t)$, because $\lambda$ is left-continuous. Furthermore, $\mu\left(s_{0}\right) \leq \alpha_{n}|x|^{n}$. Otherwise $\mu\left(s_{0}\right)>\alpha_{n}|x|^{n}$ and hence, because $\mu\left(s_{0}\right)=\lambda\left(s_{0}^{+}\right)$, there would exist an $s, s>s_{0}$, such that $\mu\left(s_{0}\right) \geq \lambda(s)>\alpha_{n}|x|^{n}$. Altogether $x \in B_{s_{0}} \backslash A_{s_{0}}=C_{s_{0}} \subset \bigcup_{s \geq t} C_{s}$.

1.4.3. Lemma. If $u \in C^{0}\left(\mathbb{R}^{n}\right)$, then $u^{*-1}\{t\}=C_{t}$ for all $t \geq 0$.

Proof. Fix $0<t \leq M$. Let $x \in C_{t}$, i.e., $\lambda(t) \geq \alpha_{n}|x|^{n} \geq \mu(t)$. The definition of $u^{*}$ gives $u^{*}(x) \leq t$. Since $\mu(s)>\lambda(t) \geq \alpha_{n}|x|^{n}$ for any $s<t$ (by Lemma 1.4.1), it follows that $u^{*}(x) \geq s$ for all $s<t$, and hence $u^{*}(x) \geq t$. Therefore $x \in u^{*-1}\{t\}$.

If $x \in u^{*-1}\{t\}$, then $\mu(t) \leq \alpha_{n}|x|^{n}$ by the definition of $u^{*}$ and the rightcontinuity of $\mu$. Also, $\mu(s)>\alpha_{n}|x|^{n}$ for any $s<t$. The relation $\lambda(t)=\mu\left(t^{-}\right)$ therefore yields $\lambda(t) \geq \alpha_{n}|x|^{n}$. Hence $x \in C_{t}$ as required.

1.4.4. Theorem. If $u \in C^{0}\left(\mathbb{R}^{n}\right)$, then $u^{*} \in C^{0}\left(\mathbb{R}^{n}\right)$.

Proof. By Lemmas 1.4.2 and 1.4.3, for any $t \geq 0$,

$$
u^{*-1}(t, \infty)=\bigcup_{s>t} u^{*-1}\{s\}=\bigcup_{s>t} C_{s}=A_{t}
$$

is open, and

$$
u^{*-1}[t, \infty)=\bigcup_{s \geq t} u^{*-1}\{s\}=\bigcup_{s \geq t} C_{s}=B_{t}
$$

is closed.

1.4.5. Corollary. $\tilde{u}$ is continuous on $[0, \infty)$ if $u \in C^{0}\left(\mathbb{R}^{n}\right)$.

1.5. $u \in C^{0}\left(\mathbb{R}^{n}\right)$ implies $u^{*} \in W^{1,1}\left(\mathbb{R}^{n}\right)$ if $\mu^{\prime} \neq 0$ a.e.

1.5.1. Lemma. If $u \in C^{0}\left(\mathbb{R}^{n}\right)$, then $\left.\tilde{u} \circ\left(\mu / \alpha_{n}\right)^{1 / n}\right|_{[0, M]}=1_{[0, M]}=$ identity $\left.\right|_{[0, M]}$. Proof. By virtue of Lemma 1.4.1, $\mu$ is strictly decreasing on $[0, M]$. Thus, for $0<t \leq M$,

$$
\begin{aligned}
\tilde{u}\left(\left(\mu(t) / \alpha_{n}\right)^{1 / n}\right) & =\sup \left\{s: \mu(s)>\alpha_{n}\left(\left(\mu(t) / \alpha_{n}\right)^{1 / n}\right)^{n}\right\} \\
& =\sup \{s: \mu(s)>\mu(t)\}=t,
\end{aligned}
$$

and $\tilde{u}\left(\left(\mu(0) / \alpha_{n}\right)^{1 / n}\right)=0$ by definition of $\tilde{u}$.

1.5.2. Proposition. If $u \in C^{0}\left(\mathbb{R}^{n}\right)$ and $\mu^{\prime} \neq 0$ almost everywhere on $[0, M]$, then $\tilde{u}$ is absolutely continuous on $[0, \infty)$.

Proof. It is sufficient to show that $N \subset[0, \infty)$ and $\mathscr{L}(N)=0$ implies $\mathscr{L}(\tilde{u}(N))=0$. Put

$$
\Omega=\left\{r \geq 0: \operatorname{card}\left(\tilde{u}^{-1}\{\tilde{u}(r)\}\right)=1\right\} .
$$


Then $\left.\tilde{u}\right|_{\Omega}$ is one-to-one. Since $\tilde{u}$ is monotone, the complement $C=[0, \infty) \backslash \Omega$ is a countable union of disjoint intervals on each of which $\tilde{u}$ is constant. Furthermore,

$$
\left.\left(\mu / \alpha_{n}\right)^{1 / n} \circ \tilde{u}\right|_{\Omega}=1_{\Omega} .
$$

Let $N \subset[0, \infty)$ be a null-set. Then $T=\tilde{u}(N)=\tilde{u}(N \cap \Omega) \cup \tilde{u}(N \cap C)$ and, by (7),

$$
\begin{aligned}
\left(\mu / \alpha_{n}\right)^{1 / n}(T) & =\left(\left(\mu / \alpha_{n}\right)^{1 / n}(\tilde{u}(N \cap \Omega))\right) \cup\left(\left(\mu / \alpha_{n}\right)^{1 / n}(\tilde{u}(N \cap C))\right) \\
& =(N \cap \Omega) \cup\left(\left(\mu / \alpha_{n}\right)^{1 / n}(\tilde{u}(N \cap C))\right) .
\end{aligned}
$$

But $\mathscr{L}(N \cap \Omega)=0$ and $\tilde{u}(N \cap C)$ is countable yield $\mathscr{L}\left(\left(\mu / \alpha_{n}\right)^{1 / n}(T)\right)=0$. Because $\mu$ is one-to-one on $[0, M], T=\mu^{-1}(\mu(T))$ is therefore measurable. Furthermore,

$$
0 \leq-\int_{T} \mu^{\prime}(t) d t \leq \mathscr{L}(\mu(T))=0 .
$$

Since $\mu^{\prime}<0$ a.e. on $[0, M]$ and $T=\tilde{u}(N) \subset[0, M]$, we conclude that $\mathscr{L}(\tilde{u}(N))=0$.

1.5.3. Theorem. If $u \in C^{0}\left(\mathbb{R}^{n}\right)$ and $\mu^{\prime} \neq 0$ a.e. on $[0, M]$, then $u^{*} \in$ $W^{1,1}\left(\mathbb{R}^{n}\right)$.

1.5.4. Remark. If $u \in C^{n}\left(\mathbb{R}^{n}\right)$, then the condition $\mu^{\prime} \neq 0$ a.e. on $[0, M]$ is satisfied.

Proof. Let $R$ be the set of all regular values of $u$ in the interval $(0, M)$. By Sard's Theorem, the complement of $R$ in $(0, M)$ has measure zero. Moreover, $R$ is an open set. Let $t \in R$ and take $t^{\prime}>t$ such that $\left[t, t^{\prime}\right]$ is contained in $R$. Then

$$
\mu(t)-\mu\left(t^{\prime}\right)=\int_{u^{-1}\left(t, t^{\prime}\right\}} p d \mathscr{L}^{n}=\int_{t}^{t^{\prime}} \int_{u^{-1}\{s\}} p|D u|^{-1} d \mathscr{H}^{n-1} d s
$$

by the co-area formula for $C^{n}$-functions in $\mathbb{R}^{n}$ (see [S] for an elementary, classical proof, [Z], and also Remark 1.5.5 below). This implies that

$$
\mu^{\prime}(t)=-\int_{u^{-1}\{t\}} p|D u|^{-1} d \mathscr{P}^{n-1}
$$

for a.e. $t$. Hence $\mu^{\prime}(t)<0$ a.e. on $(0, M)$.

1.5.5. Remark. In order to apply the co-area formula, one changes the density distribution $p$ to a Borel measurable function by writing $p=\sum_{k=1}^{\infty} \frac{1}{k} \chi_{A_{k}}$ with $\mathscr{L}^{n}$-measurable sets $A_{k}$, and replacing $A_{k}$ by Borel sets $B_{k}$ with $B_{k} \supset A_{k}$ and $\mathscr{L}^{n}\left(B_{k} \backslash A_{k}\right)=0$.

1.6. $u \in W^{1,1}\left(\mathbb{R}^{n}\right)$ implies $u^{*} \in W^{1,1}\left(\mathbb{R}^{n}\right)$. The fact that $\lambda(s)<\mu(t)$ for all $0 \leq t<M, t<s$ (Lemma 1.4.1) was crucial in proving the regularity properties of $u^{*}$ (provided that $u \in C^{0}\left(\mathbb{R}^{n}\right)$ and $\mu^{\prime} \neq 0$ a.e. on $[0, M]$ ). Now we shall show that this remains true if $u \in W^{1,1}\left(\mathbb{R}^{n}\right)$. Analogues of Lemma 1.4.2-Theorem 1.5.3 will then hold. First recall: 
1.6.1. Remark. A bounded measurable set $A$ has finite perimeter $\mathscr{P}(A)=$ $\left\|D \chi_{A}\right\|$ if its characteristic function $\chi_{A}$ is of bounded variation. The following isoperimetric inequality follows by approximation from a corresponding Sobolev inequality $[\mathrm{EG}, \mathrm{Z}]$ :

$$
\mathscr{L}^{n}(A)^{(n-1) / n} \leq C(n) \mathscr{P}(A) .
$$

1.6.2. Lemma. If $u \in W^{1,1}\left(\mathbb{R}^{n}\right), 0 \leq t<M=\operatorname{ess} \sup u$, and $t<s$, then $\mathscr{L}^{n}\left(u^{-1}(t, s)\right) \neq 0$.

Proof. Assume by contradiction that $\mathscr{L}^{n}\left(u^{-1}(t, s)\right)=0$ for some $t, s, 0 \leq$ $t<M, t<s$. Then, for any test function $g \in C_{0}^{\infty}\left(\mathbb{R}^{n}\right)$,

$$
\begin{aligned}
\int_{\mathbb{R}^{n}} D_{i} u g d \mathscr{L}^{n} & =\int_{u^{-1}(-\infty, t]} D_{i} u g d \mathscr{L}^{n}+\int_{u^{-1}[s, \infty)} D_{i} u g d \mathscr{L}^{n} \\
& =\int_{\mathbb{R}^{n}} \max (t-u, 0) D_{i} g d \mathscr{L}^{n}+\int_{\mathbb{R}^{n}} \max (s-u, 0) D_{i} g d \mathscr{L}^{n} \\
& =\int_{u^{-1}(-\infty, t]}(t-u) D_{i} g d \mathscr{L}^{n}+\int_{u^{-1}[s, \infty)}(s-u) D_{i} g d \mathscr{L}^{n} .
\end{aligned}
$$

On the other hand,

(9)

$$
\begin{aligned}
\int_{\mathbb{R}^{n}} D_{i} u g d \mathscr{L}^{n} & =-\int_{\mathbb{R}^{n}}(u-t) D_{i} g d \mathscr{L}^{n} \\
& =-\int_{u^{-1}(-\infty, t]}(u-t) D_{i} g d \mathscr{L}^{n}-\int_{u^{-1}[s, \infty)}(u-t) D_{i} g d \mathscr{L}^{n} .
\end{aligned}
$$

Since $t \neq s$, it follows from (8) and (9) that

$$
\int_{u^{-1}[s, \infty)} D_{i} g d \mathscr{L}^{n}=0 .
$$

Hence $\mathscr{P}(F)=0, F=u^{-1}[s, \infty)$. The application of the isoperimetric inequality $\left(\mathscr{L}^{n}(F)\right)^{(n-1) / n} \leq C(n) \mathscr{P}(F)$ yields

$$
\mathscr{L}^{n}\left(u^{-1}(t, \infty)\right)=\mathscr{L}^{n}\left(u^{-1}(t, s)\right)+\mathscr{L}^{n}\left(u^{-1}[s, \infty)\right)=0,
$$

contradicting the definition of $M$.

1.6.3. Lemma. If $u \in W^{1,1}\left(\mathbb{R}^{n}\right)$, then $\lambda(s)<\mu(t)$ for any $0 \leq t<M, t<s$. Moreover, $\mu$ and $\lambda$ are stricly decreasing functions on $[0, M)$.

In order to prove that $\mu^{\prime} \neq 0$ a.e., we wish to apply a co-area formula for Sobolev functions. The general version which is stated in [BZ] is taken from Federer's section on normal currents of dimension $n$ in $\mathbb{R}^{n}[\mathrm{~F}$, Theorem 4.5.9 (in particular statement (14))]. The proof of the next lemma would then require further discussion of reduced boundary, measure-theoretic boundary, the generalized Gauss-Green theorem, and an isometric inequality involving measure-theoretic boundary (like [F, Theorem 4.5.9, statement (31)] or [B]). However, we wish to keep things as elementary as possible and recall:

1.6.4. Remark. Assume that $f \in \mathrm{BV}\left(\mathbb{R}^{n}\right)$. Then the variation measure of $f$ is given by

$$
\|D f\|(U)=\sup \left\{\int_{\mathbb{R}^{n}} f \operatorname{div} h d \mathscr{L}^{n}: h \in C_{0}^{\infty}\left(\mathbb{R}^{n}, \mathbb{R}^{n}\right),|h| \leq 1\right\}
$$


for any open set $U$ in $\mathbb{R}^{n}$. $\|D f\|$ is a Radon measure given by the Riesz representation theorem. Denote the perimeter measure of a set $E$ with finite perimeter by

$$
\mathscr{P}(E)=\mathscr{P}(E, U)=\left\|D \chi_{E}\right\|(U) .
$$

Let $E_{t}=f^{-1}(t, \infty)$. Then the co-area formula for BV-functions (see [EG] or [Z]) states that $\mathscr{P}\left(E_{t}, U\right)$ is a Lebesgue measurable function of $t$, finite a.e., and

$$
\|D f\|(U)=\int_{\mathbb{R}} \mathscr{P}\left(E_{t}, U\right) d t .
$$

Hence, for any nonnegative Borel measurable function $g$,

$$
\int_{\mathbb{R}^{n}} g d\|D f\|=\int_{-\infty}^{+\infty} \int_{\mathbb{R}^{n}} g d \mathscr{P}\left(E_{t}\right) d t
$$

If $f$ is a Sobolev function of class $W^{1,1}$, then $\|D f\|$ is absolutely continuous with respect to the Lebesgue measure $\mathscr{L}^{n}$ and

$$
\|D f\|(U)=\int_{U}|D f| d \mathscr{L}^{n} .
$$

Therefore, our co-area formula for Sobolev functions is

$$
\int_{\mathbb{R}^{n}} g|D f| d \mathscr{L}^{n}=\int_{-\infty}^{+\infty} \int_{\mathbb{R}^{n}} g d \mathscr{P}\left(E_{t}\right) d t
$$

for any nonnegative Borel function $g$.

1.6.5. Lemma. $u \in W^{1,1}\left(\mathbb{R}^{n}\right)$ implies $\mu^{\prime} \neq 0$ a.e. on $[0, M]$.

Proof. By Remark 1.5.5 we may assume w.l.o.g. that $p$ is Borel measurable, and we may also choose a Borel measurable representative for $u$. Let $C=$ $\{x: D u=0\}$, and note that $\mathscr{P}\left(E_{t}, C\right)=0$ a.e. by the co-area formula, which then yields

$$
\begin{aligned}
\mu(t)-\mu\left(t^{\prime}\right) & =\int_{u^{-1}\left(t, t^{\prime}\right]} p d \mathscr{L}^{n} \\
& =\mathscr{M}^{n}\left(u^{-1}\left(t, t^{\prime}\right] \cap C\right)+\int_{u^{-1}\left(t, t^{\prime}\right]}\left(p|D u|^{-1}\right)|D u| d \mathscr{L}^{n} \\
& =\mathscr{M}^{n}\left(u^{-1}\left(t, t^{\prime}\right] \cap C\right)+\int_{t}^{t^{\prime}} \int_{\mathbb{R}^{n}} p|D u|^{-1} d \mathscr{P}\left(E_{s}\right) d s
\end{aligned}
$$

Hence

$$
-\mu^{\prime}(t) \geq \int_{\mathbb{R}^{n}} p|D u|^{-1} d \mathscr{P}\left(E_{t}\right)
$$

for a.e. $t$. From Lemma 1.6.2 and the isoperimetric inequality given in Remark 1.6.1, we have

$$
0<\left(\mathscr{L}^{n}\left(E_{t}\right)\right)^{(n-1) / n} \leq C(n) \mathscr{P}\left(E_{t}\right)
$$

and, since $\mathscr{L}^{n}(\{x:|D u|=\infty\})=0$, it follows that $\mathscr{P}\left(E_{t},\{x:|D u|=\infty\}\right)=0$ for a.e. $t$ by the co-area formula. Hence $-\mu^{\prime}(t)>0$ a.e. as stated.

With the aid of these lemmas, we can follow the proofs of Lemma 1.4.2Proposition 1.5.2 to obtain 
1.6.6. Proposition. Suppose that $u \in W^{1,1}\left(\mathbb{R}^{n}\right)$. Then

(i) $u^{*-1}\{t\}=C_{t}$ for all $t \geq 0 \quad\left(A_{\infty}=B_{\infty}=C_{\infty}=\varnothing\right.$ if $\left.M=+\infty\right)$.

(ii) $u^{*}$ is continuous on $\mathbb{R}^{n} \backslash\{0\}$ and continuous on $\mathbb{R}^{n}$ if $u \in L^{\infty}$.

(iii)

$$
\left.\tilde{u} \circ\left(\mu / \alpha_{n}\right)^{1 / n}\right|_{[0, M]}=1_{[0, M]} .
$$

(iv) $\tilde{u}$ is absolutely continuous on $(0, \infty)$ and absolutely continuous on $[0, \infty)$ if $u \in L^{\infty}$.

1.6.7. Theorem. If $u \in W^{1,1}\left(\mathbb{R}^{n}\right) \cap L^{\infty}$, then $u^{*} \in W^{1,1}\left(\mathbb{R}^{n}\right)$.

Proof. Proposition 1.6.6(iv) implies that the gradient of $u^{*}, D u^{*}$, exists in the distributional sense, and by Theorem 1.3.1,

$$
\int_{\mathbb{R}^{n}}\left|D u^{*}\right| d \mathscr{L}^{n}=\left\|D u^{*}\right\|<\infty .
$$

Also, from the proof of Theorem 1.3.1:

1.6.8. Theorem. If $u \in W^{1,1}\left(\mathbb{R}^{n}\right)$, then $u^{*} \in W_{\mathrm{loc}}^{1,1}\left(\mathbb{R}^{n} \backslash\{0\}\right)$.

As a final remark we note that under the conditions of Theorems 1.5 .3 or 1.6.8, the following co-area formula for $u^{*}$ is true (see [S] for an elementary proof, or $[\mathrm{F}, \mathrm{BZ}])$ :

1.6.9. Remark. If $g$ is a nonnegative Borel measurable function on $[0, \infty)$, then

$$
\int_{\mathbb{R}^{n}} g\left(u^{*}(x)\right)\left|D u^{*}(x)\right| d \mathscr{L}^{n}=\int_{0}^{\infty} g(t) \int_{u^{*-1}\{t\}} d \mathscr{H}^{n-1} d t .
$$

1.7. Absolute continuity of $\mu$. Suppose in this section that $u \in W^{1,1}\left(\mathbb{R}^{n}\right)$.

1.7.1. Proposition. Let $C^{*}=\left\{x: D u^{*}(x)=0\right\}$. Then $\mu$ is absolutely continuous on $(0, M)$ if and only if

$$
\mathscr{L}^{n}\left(u^{*-1}(0, M) \cap C^{*}\right)=0 .
$$

Proof. We may assume w.l.o.g. that $u^{*} \in W^{1,1}\left(\mathbb{R}^{n}\right)$ by otherwise cutting off $u$. Suppose that $\mathscr{L}^{n}\left(u^{*-1}(0, M) \cap C^{*}\right)=0$. The co-area formula then implies that for all $t, 0<t<M$ (see also Remark 1.7.3 below),

$$
\begin{aligned}
\mu(0)-\mu(t) & =\mathscr{L}^{n}\left(u^{*-1}(0, t) \cap C^{*}\right)+\int_{u^{*-1}(0, t)}\left|D u^{*}\right|^{-1}\left|D u^{*}\right| d \mathscr{L}^{n} \\
& =\int_{0}^{t} \int_{\mathbb{R}^{n}}\left|D u^{*}\right|^{-1} d \mathscr{P}\left(E_{s}\right) d s=\int_{0}^{t} \int_{u^{*-1}\{s\}}\left|D u^{*}\right|^{-1} d \mathscr{H}^{n-1} d s,
\end{aligned}
$$

from which the absolute continuity of $\mu$ follows.

Suppose that $\mu$ is absolutely continuous. The previous application of the coarea formula shows that the function $\mathscr{L}^{n}\left(u^{*-1}(0, t) \cap C^{*}\right)$ is then absolutely continuous. Hence if $N \subset(0, M)$ is a null set, then $\mathscr{L}^{n}\left(u^{*-1}(N) \cap C^{*}\right)=0$. By invoking the co-area formula again, it follows that

$$
\begin{aligned}
0 & =\int_{C^{*} \cap u^{*-1}(0, M)}\left|D u^{*}\right| d \mathscr{L}^{n}=\int_{0}^{M} \int_{\mathbb{R}^{n}} \chi_{C^{*}} d \mathscr{P}\left(E_{s}\right) d s \\
& =\int_{0}^{M} \int_{u^{*-1}\{s\} \cap C^{*}} d \mathscr{H}^{n-1} d s,
\end{aligned}
$$


or equivalently,

$$
0=\int_{\widetilde{C} \cap \tilde{u}^{-1}(0, M)}\left|u^{\prime}(r)\right| d r=\int_{0}^{M} \operatorname{card}\left(\tilde{u}^{-1}\{s\} \cap \tilde{C}\right) d s,
$$

where $\tilde{C}=\left\{r: \tilde{u}^{\prime}(r)=0\right\}$, which implies the Sard type fact that

$$
\mathscr{H}^{n-1}\left(u^{*-1}\{s\} \cap C^{*}\right)=0 \text { for a.e. } s \text {. }
$$

This in turn implies that $u^{*-1}\{s\} \cap C^{*}=\varnothing$ a.e. Therefore, $u^{*-1}(0, M) \cap C^{*}=$ $u^{*-1}(N) \cap C^{*}$ for a null set $N \subset(0, M)$, and hence $\mathscr{L}^{n}\left(u^{*-1}(0, M) \cap C^{*}\right)=0$ by the absolute continuity of $\mathscr{L}^{n}\left(u^{*-1}(0, t) \cap C^{*}\right)$.

The proof of the next assertion is similar.

1.7.2. Lemma. $\mu$ is absolutely continuous on $(0, M)$ if

$$
\mathscr{L}^{n}\left(u^{-1}(0, M) \cap\{x: D u(x)=0\}\right)=0 .
$$

1.7.3. Remark. In order to facilitate an elementary application of the co-area formula, note that there exists a nonnegative Borel measurable function $\phi$ such that

$$
\left|D u^{*}(x)\right|=\phi\left(u^{*}(x)\right) \text { a.e. }
$$

To prove this fact note that the difference quotients $\Delta_{h} \tilde{u}(r)=(\tilde{u}(r+h)-\tilde{u}(r)) / h$ are $\mathscr{A}(\tilde{u})$-measurable, where $\mathscr{A}(\tilde{u})=\left\{\tilde{u}^{-1}(B): B \in \mathscr{B}(\mathbb{R})\right\}$ is the smallest $\sigma$ algebra such that $\tilde{u}$ is measurable. Hence $D^{+} \tilde{u}(r)=\liminf \Delta_{1 / k} \tilde{u}(r) \quad(k \rightarrow \infty)$ is $\mathscr{A}(\tilde{u})$-measurable, and by a measure-theoretic lemma, there exists a Borel measurable function such that $D^{+} \tilde{u}=\phi \circ \tilde{u}$. Therefore, $\tilde{u}^{\prime}(r)=\phi(\tilde{u}(r))$ a.e.

\section{Minimal REARRANGEMENTS AND REgULARITY PROPERTIES ON $\mathbb{R}^{2}$}

2.1. Carleman's isoperimetric inequality. Carleman [C] proved the classical isoperimetric inequality for domains on a minimal surface by viewing the Weierstrass formulae as defining a conformal map from the minimal surface onto a simply-connected plane Riemann surface and thus yielding a conformal map onto the unit disc $\mathbb{D}$. The isoperimetric inequality follows then from the inequality

$$
4 \pi \int_{0}^{1} \int_{0}^{2 \pi}\left|f\left(r e^{i \theta}\right)\right|^{2} r d r d \theta \leq\left(\int_{0}^{2 \pi}\left|f\left(e^{i \theta}\right)\right| d \theta\right)^{2}
$$

for analytic functions in the closed unit disc $\overline{\mathbb{D}}$. Carleman's proof of this inequality follows by choosing $f$ to be free of zeros and then expanding $f$ into a power series. The proof is valid in the following situation which, in Lozinski's formulation [L], reads as:

2.1.1. Carleman's inequality in $\mathbb{D}$. The inequality (12) is valid if $f$ belongs to the Hardy space $H^{1}$ of analytic functions in $\mathbb{D}$ which satisfy

$$
\sup _{0 \leq r<1} \int_{0}^{2 \pi}\left|f\left(r e^{i \theta}\right)\right| d \theta<\infty .
$$

The radial limit $f\left(e^{i \theta}\right):=\lim _{r \rightarrow 1}-f\left(r e^{i \theta}\right)$ on the right-hand side of (12) exists for almost every $\theta, 0 \leq \theta \leq 2 \pi$. If $f \not \equiv 0$ on $\mathbb{D}$, then equality takes place if 
and only if $f(z)=F^{\prime}(z)$ for all $z$ in $\mathbb{D}$, where $F(z)=(a z+b) /(c z+d)$ is a linear fractional transformation which is analytic on $\overline{\mathbb{D}}$. Here $a, b, c$, and $d$ are complex numbers with $a d-b c \neq 0$.

Let $p \in C^{0}(\bar{D})$ be a positive function such that $\log p$ is subharmonic in a simply-connected domain $D$. Consider the inequality

$$
4 \pi \int_{D} p d \mathscr{L}^{2} \leq\left(\int_{\partial D} \sqrt{p} d \mathscr{H}^{1}\right)^{2} .
$$

Since $D$ is a Dirichlet domain, we can replace $\frac{1}{2} \log p$ by its least harmonic majorant, which is of class $C^{2}(D) \cap C^{0}(\bar{D})$, and complete to an analytic function. If the domain $D$ is bounded by a rectifiable Jordan curve $\Gamma$, then the inequality (13) follows from Carleman's inequality in $\mathbb{D}$ by invoking the Riemann mapping theorem, and is therefore equivalent to it (the boundary regularity is readily verified; see, e.g., Huber [HU]):

2.1.2. Carleman's inequality in $D$. Suppose that $D$ is a simply connected domain in $\mathbb{R}^{2}$ which is bounded by a rectifiable Jordan curve $\Gamma$ and let $p \in C^{0}(\bar{D})$ be such that $\log p$ is subharmonic on $D$. Then

$$
4 \pi \iint_{D} p d x d y \leq\left(\int_{\Gamma} \sqrt{p} d s\right)^{2} .
$$

If $p \not \equiv 0$ on $D$, then equality takes place if and only if $p(z)=\left|F^{\prime}(z)\right|^{2}$, where $F$ is a one-to-one conformal map from $D$ onto a disk.

2.1.3. Remark. The mapping $F$ has a unique topological extension to $\bar{D}$. A reference is Behnke-Sommer [BS, Satz 43, p. 369] (a "geschlossene einfache Kurve" is a Jordan curve).

2.1.4. Remark. Carleman's inequality extends to the situation where $D$ is bounded by finitely many disjoint Jordan curves which are contained in a simply-connected domain $G$ in which $\log p$ is subharmonic.

Carleman's inequality will now be generalized to sets of finite perimeter.

2.1.5. Assumption. The function $p$ is positive and continuous on a simplyconnected domain $G$ in $\mathbb{R}^{2}$ such that $\log p$ is subharmonic.

2.1.6. Theorem. The following Sobolev type inequality holds for all $f \in C^{2}\left(\mathbb{R}^{2}\right)$ with compact support in $G$ :

$$
4 \pi \iint_{\mathbb{R}^{2}}|f|^{2} p d \mathscr{L}^{2} \leq\left(\iint_{\mathbb{R}^{2}}|D f| \sqrt{p} d \mathscr{L}^{2}\right)^{2}
$$

Proof. For $t \in \mathbb{R}$ let

$$
f_{t}(x)= \begin{cases}f(x), & f(x) \leq t \\ t, & f(x)>1\end{cases}
$$

and consider the function $F(t)=\left(\int_{\mathbb{R}^{2}}\left|f_{t}\right|^{2} d \mathscr{M}^{2}\right)^{1 / 2}$. For all $h \geq 0$ estimate

$$
0 \leq F(t+h)-F(t) \leq\left(\int_{\mathbb{R}^{2}}\left|f_{t+h}-f_{t}\right|^{2} d \mathscr{M}^{2}\right)^{1 / 2} \leq h\left(\mathscr{M}^{2}\left(E_{t}\right)\right)^{1 / 2}
$$


where $E_{t}=f^{-1}(t, \infty)$. The function $F(t)$ is therefore Lipschitz continuous with $0 \leq F^{\prime}(t) \leq\left(\mathscr{K}^{2}\left(E_{t}\right)\right)^{1 / 2}$. By integrating with respect to $t$ from $-\infty$ to $+\infty$ (in fact only over a finite interval), we obtain

$$
\begin{aligned}
\left(\int_{\mathbb{R}^{2}}|f|^{2} d \mathscr{M}^{2}\right)^{1 / 2} & =\int_{-\infty}^{+\infty} F^{\prime}(t) d t \leq \int_{-\infty}^{+\infty}\left(\mathscr{M}^{2}\left(E_{t}\right)\right)^{1 / 2} d t \\
& \leq 2 \sqrt{\pi} \int_{-\infty}^{+\infty} \int_{f^{-1}\{t\}} \sqrt{p} d \mathscr{H}^{1} d t=2 \sqrt{\pi} \int_{\mathbb{R}^{2}}|D f| \sqrt{p} d \mathscr{L}^{2}
\end{aligned}
$$

by the co-area formula for $C^{2}$-functions in $\mathbb{R}^{2}$ (see [S] or [Z]) and by Carleman's inequality (14), which, by Remark 2.1.4, remains true in our situation: Since $f \in C^{2}\left(\mathbb{R}^{2}\right)$, Sard's theorem states that $f^{-1}\{t\}$ is a closed, compact $C^{2}$ submanifold of $\mathbb{R}^{2}$ for almost every value $t$, and $u^{-1}\{t\}=\partial E_{t}$ is therefore the union of finitely many disjoint, rectifiable Jordan curves.

2.1.7. Theorem. If $f \in \mathrm{BV}\left(\mathbb{R}^{2}\right)$ with compact support in $G$, then

$$
4 \pi \int_{\mathbb{R}^{2}}|f|^{2} p d \mathscr{L}^{2} \leq\left(\int_{\mathbb{R}^{2}} \sqrt{p} d\|D f\|\right)^{2} .
$$

Proof. Let $\left\{f_{k}\right\}_{k=1}^{\infty}$ be a sequence of functions in $C_{0}^{\infty}(U)$ such that $f_{k} \rightarrow f$ in $L^{1}$ and $\left\|D f_{k}\right\|(U) \rightarrow\|D f\|(U)$. Then

$$
\left\|D f_{k}\right\| \rightarrow\|D f\|
$$

(weakly) in the sense of measures, and therefore

$$
\int_{\mathbf{R}^{2}} \sqrt{p} d\left\|D f_{k}\right\| \rightarrow \int_{\mathbb{R}^{2}} \sqrt{p} d\|D f\|=\int_{\mathbb{R}^{2}} \sqrt{p}|D f| d \mathscr{L}^{2} .
$$

By specializing $f=\chi_{E}$ one obtains

2.1.8. Theorem. Suppose that $E$ is a bounded set of finite perimeter which is contained in $G$. Then the following isoperimetric inequality holds:

$$
4 \pi \int_{E} p d \mathscr{L}^{2} \leq\left(\int_{\mathbb{R}^{2}} \sqrt{p} d \mathscr{P}(E)\right)^{2} .
$$

2.1.9. Remark. By the structure theorem for sets of finite perimeter, $\mathscr{P}(E)=$ $\left.\mathscr{H}^{1}\right|_{\partial_{\nu} E}$, where $\partial_{\nu} E$ is the reduced boundary of $E$, i.e., the subset of all points in $\partial E$ for which there exists a generalized normal.

2.2. The energy inequality $\left\|D u^{*}\right\|_{2} \leq\|D u\|_{2}$.

2.2.1. Assumption. The function $u$ is nonnegative with compact support in $G$ of class $W^{1,2}\left(\mathbb{R}^{2}\right)$.

2.2.2. Lemma. For almost all $t \geq 0$,

$$
\int_{u^{*-1}\{t\}} d \mathscr{H}^{1} \leq \int_{\mathbb{R}^{2}} \sqrt{p} d \mathscr{P}\left(E_{t}\right)
$$

Proof. By the co-area formula for sets of finite perimeter (see formula (10)), we have $\mathscr{P}\left(E_{t}\right)<\infty$ for almost every $t$. If we choose $t$ to also satisfy $u^{*-1}\{t\}=$ $\partial E_{t}^{*}$, then

$$
\int_{u^{*-1}\{t\}} d \mathscr{H}^{1}=2 \sqrt{\pi}\left(\mathscr{L}^{2}\left(E_{t}^{*}\right)\right)^{1 / 2}=2 \sqrt{\pi}\left(\mathscr{M}^{2}\left(E_{t}\right)\right)^{1 / 2} \leq \int_{\mathbb{R}^{2}} \sqrt{p} d \mathscr{P}\left(E_{t}\right)
$$

by Theorem 2.1.8. 
2.2.3. Theorem. Suppose that Assumptions 2.1.5 and 2.2.1 are satisfied, i.e., the function $p$ is positive and continuous on a simply-connected domain $G$ such that $\log p$ is subharmonic, and $u \in W^{1,2}\left(\mathbb{R}^{2}\right)$ is a nonnegative function with compact support in $G$. Then $u^{*} \in W^{1,2}\left(\mathbb{R}^{2}\right)$, and the energy inequality

$$
\int_{\mathbb{R}^{2}}\left|D u^{*}\right|^{2} d \mathscr{L}^{2} \leq \int_{\mathbb{R}^{2}}|D u|^{2} d \mathscr{L}^{2}
$$

holds.

Proof. Let $\phi$ be a nonnegative Borel function with $\phi\left(u^{*}(x)\right)=\left|D u^{*}(x)\right|$ for almost every $x$ in $\mathbb{R}^{2}$ (see Remark 1.7.3), and let

$$
\phi_{k}(t)= \begin{cases}\phi(t), & \phi(t) \leq k, \\ k, & \phi(t)>k,\end{cases}
$$

for each $k \in \mathbb{N}$. Then

$$
\begin{aligned}
\int_{\mathbb{R}^{2}}( & \left.\phi_{k}\right)^{2}\left(u^{*}(x)\right) d \mathscr{L}^{2} \leq \int_{\mathbb{R}^{2}} \phi_{k}\left(u^{*}(x)\right) \phi\left(u^{*}(x)\right) d \mathscr{L}^{2} \\
\quad & \int_{\mathbb{R}^{2}} \phi_{k}\left(u^{*}(x)\right)\left|D u^{*}(x)\right| d \mathscr{L}^{2}=\int_{0}^{\infty} \phi_{k}(t) \int_{u^{*-1}\{t\}} d \mathscr{L}^{1} d t \\
& \leq \int_{0}^{\infty} \phi_{k}(t) \int_{\mathbb{R}^{2}} \sqrt{p} d \mathscr{P}\left(E_{t}\right) d t=\int_{\mathbb{R}^{2}} \phi_{k}(u(x)) \sqrt{p}|D u| d \mathscr{L}^{2} \\
& \leq\left(\int_{\mathbb{R}^{2}}\left(\phi_{k}\right)^{2}(u(x)) p d \mathscr{L}^{2}\right)^{1 / 2}\|D u\|_{2}=\left(\int_{\mathbb{R}^{2}}\left(\phi_{k}\right)^{2}\left(u^{*}(x)\right) d \mathscr{L}^{2}\right)^{1 / 2}\|D u\|_{2}
\end{aligned}
$$

by employing the co-area formula (11), Lemma 2.2.2, the co-area formula for Sobolev functions, formula (10), and Corollary 1.2.4. Hence

$$
\int_{\mathbb{R}^{2}}\left(\phi_{k}\right)^{2}\left(u^{*}(x)\right) d \mathscr{L}^{2} \leq\|D u\|_{2}^{2}
$$

for $k=1,2, \ldots$, and the stated energy inequality (17) follows by letting $k \rightarrow \infty$.

2.3. Minimal rearrangements, the equality $\left\|D u^{*}\right\|_{2}=\|D u\|_{2}$.

2.3.1. Assumption. The energy equality $\left\|D u^{*}\right\|_{2}=\|D u\|_{2}$ holds.

2.3.2. Lemma. For almost all $t \geq 0$,

$$
\int_{u^{*-1}\{t\}} d \mathscr{H}^{1}=\int_{\mathbb{R}^{2}} \sqrt{p} d \mathscr{P}\left(E_{t}\right) .
$$

Proof. Observe that whenever $u^{*-1}\{t\} \neq \partial E_{t}^{*}$, then the value $t$ is taken by the function $u^{*}$ on a set of positive measure. Therefore, $u^{*-1}\{t\} \neq \partial E_{t}^{*}$ at most for countably many $t$ 's. The energy equality implies that all inequalities in the proof of Theorem 2.2.3 are equalities as well. In particular,

$$
\phi(t) \int_{u^{*-1}\{t\}} d \mathscr{H}^{1}=\phi(t) \int_{\mathbb{R}^{2}} \sqrt{p} d \mathscr{P}\left(E_{t}\right)
$$

for almost all $t \geq 0$. The statement follows from the fact that $\phi(t) \neq 0$ for almost all $t \in[0, M]$, as can be seen, for example, from the Sard type fact that

$$
\mathscr{H}^{1}\left(u^{*-1}\{t\} \cap C^{*}\right)=0
$$


for almost all $t$, where $C^{*}=\left\{x: D u^{*}(x)=0\right\}$, which follows by applying the co-area formula to $\tilde{u}$ (see also the proof of Proposition 1.7.1):

$$
0=\int_{\widetilde{C}}\left|\tilde{u}^{\prime}(r)\right| d r=\int_{0}^{\infty} \operatorname{card}\left(\tilde{u}^{-1}\{t\} \cap \widetilde{C}\right) d t,
$$

where $\widetilde{C}=\left\{r: \tilde{u}^{\prime}(r)=0\right\}$, which implies that $\tilde{u}^{-1}\{t\} \cap \widetilde{C}=\varnothing$ for almost all $t$.

For the proof of the next statement recall:

2.3.3. Remark. By the proof of the Riesz representation theorem, if $f \in$ $\mathrm{BV}\left(\mathbb{R}^{n}\right)$ and $g \in C_{0}^{\infty}\left(\mathbb{R}^{n}, \mathbb{R}_{0}^{+}\right)$, then

$$
\int_{\mathbb{R}^{n}} g d\|D f\|=\|D f\|(g)=\sup \left\{\int_{\mathbb{R}^{n}} f \operatorname{div} h d \mathscr{L}^{n}: h \in C_{0}^{\infty}\left(\mathbb{R}^{n}, \mathbb{R}^{n}\right),|h| \leq g\right\} .
$$

2.3.4. Lemma. $E_{0}$ is a set of finite perimeter, and

$$
\int_{\partial E_{0}^{*}} d \mathscr{H}^{1}=\int_{\mathbb{R}^{2}} \sqrt{p} d \mathscr{P}\left(E_{0}\right)
$$

Proof. Let $\left\{t_{k}\right\}_{k=1}^{\infty}$ be a sequence of $t$ 's satisfying Lemma 2.3.2 and such that $t_{k} \searrow 0$. By changing $p$ outside $E_{0}$ to a continuous function with compact support in $\mathbb{R}^{2}$, it follows for all $h \in C_{0}^{\infty}\left(\mathbb{R}^{2}, \mathbb{R}^{2}\right),|h| \leq \sqrt{p}$, that

$$
\begin{aligned}
\int_{E_{0}} \operatorname{div} h d \mathscr{L}^{2} & =\lim _{k \rightarrow \infty} \int_{E_{t_{k}}} \operatorname{div} h d \mathscr{L}^{2} \\
& \leq \liminf _{k \rightarrow \infty}\left(\mathscr{P}\left(E_{t_{k}}\right)\right)(\sqrt{p})=\liminf _{k \rightarrow \infty} \int_{\mathbb{R}^{2}} \sqrt{p} d \mathscr{P}\left(E_{t_{k}}\right) .
\end{aligned}
$$

Since $\left\{P\left(E_{t_{k}}\right)\right\}$ is bounded, $E_{0}$ is a set of finite perimeter and the lower semicontinuity

$$
\int_{\mathbb{R}^{2}} \sqrt{p} d \mathscr{P}\left(E_{0}\right)=\left(\mathscr{P}\left(E_{0}\right)\right)(\sqrt{p}) \leq \liminf _{k \rightarrow \infty} \int_{\mathbb{R}^{2}} \sqrt{p} d \mathscr{P}\left(E_{t_{k}}\right)
$$

follows. By Lemma 2.3.2,

$$
\begin{aligned}
\int_{\mathbb{R}^{2}} \sqrt{p} d \mathscr{P}\left(E_{0}\right) & \leq \liminf _{k \rightarrow \infty} \int_{\mathbb{R}^{2}} \sqrt{p} d \mathscr{P}\left(E_{t_{k}}\right) \\
& =\liminf _{k \rightarrow \infty} 2 \sqrt{\pi}\left(\mathscr{M}^{2}\left(E_{t_{k}}\right)\right)^{1 / 2}=2 \sqrt{\pi}\left(\mathscr{M}^{2}\left(E_{0}\right)\right)^{1 / 2} .
\end{aligned}
$$

The isoperimetric inequality (15) from Theorem 2.1 .8 , applied to $E_{0}$, is the required reverse inequality.

\subsection{Reduction to the case of a homogeneous density distribution.}

2.4.1. Assumption. $u \in W_{0}^{1,2}(D)$, with $D=E_{0}=u^{-1}(0, \infty)$ an open set (w.l.o.g.) which is bounded by finitely many disjoint rectifiable Jordan curves.

The following assertion follows from Lemma 2.3.4 and Carleman's Inequality 2.1.2 in combination with Remarks 2.1.3 and 2.1.4. 
2.4.2. Lemma. There is a one-to-one conformal map $\widetilde{F}$ from $D$ onto a disk $\mathbb{D}(p)$ with $p=\left|\widetilde{F}^{\prime}\right|^{2}$ on $D$. In particular, $D$ is simply connected, $F$ extends to a topological map from $\bar{D}$ onto $\overline{\mathbb{D}}$, and $\log p$ is a harmonic function on $D$.

2.4.3. Lemma. Define $v: \mathbb{R}^{2} \rightarrow \mathbb{R}_{0}^{+}$by

$$
v(y)= \begin{cases}u\left(\widetilde{F}^{-1}(y)\right) & \text { if } y \in \mathbb{D}(p), \\ 0 & \text { otherwise. }\end{cases}
$$

Then $v \in W_{0}^{1,2}(\mathbb{D}(p))$, and $u^{*}=v^{*}$, which is the spherical symmetric rearrangement of $v$ with respect to the Lebesgue measure $\mathscr{L}^{2}$.

Proof. For $t \geq 0$ let $E_{v, t}=\{y: v(y)>t\}$. Clearly $E_{v, t}=F\left(E_{t}\right)$ and $\mathscr{L}^{2}\left(E_{v, t}\right)=\mathscr{L}^{2}\left(E_{t}^{*}\right)$. This implies that $u^{*}=v^{*}$, because they are both continuous functions on $\mathbb{R}^{2} \backslash\{0\}$ by Proposition 1.6.6, statement (ii).

In order to apply the theory of symmetric rearrangements with respect to the Lebesgue measure $\mathscr{L}^{2}$ to the function $v$, we impose the following (compare $\S 1.7$ for an equivalent characterization or for a more stringent condition):

2.4.4. Assumption. $\mathscr{L}^{2}\left(u^{*-1}(0, M) \cap\left\{x: D u^{*}(x)=0\right\}\right)=0$, with $M=$ $\sup u^{*}=\sup u$.

The main result is then the following:

2.4.5. Theorem. Suppose that Assumptions 2.1.5, 2.2.1, 2.4.1, and 2.4.4 are satisfied, i.e., the function $p$ is positive and continuous on a simply-connected domain $G$ in $\mathbb{R}^{2}$ such that $\log p$ is subharmonic, $u \in W_{0}^{1,2}(D)$ is a positive function a.e. on an open set $D$ which is compactly contained in $G$ and bounded by finitely many disjoint rectifiable Jordan curves, and $\mathscr{L}^{2}\left(u^{*-1}(0, M) \cap\left\{x: D u^{*}(x)\right.\right.$ $=0\})=0 \quad(M=$ ess $\sup u)$. Then the energy equality

$$
\int_{\mathbb{R}^{2}}\left|D u^{*}\right|^{2} d \mathscr{L}^{2}=\int_{\mathbb{R}^{2}}|D u|^{2} d \mathscr{L}^{2}
$$

holds if and only if $u=u^{*} \circ F$ a.e., where $F$ is a one-to-one conformal map of $D$ onto $D^{*}$, which is the disk centered at 0 with $\mathscr{M}^{2}(D)=\mathscr{L}^{2}\left(D^{*}\right)$, with $p=\left|F^{\prime}\right|^{2}$. In particular, $D$ is simply connected, the function $F$ extends to a topological map from $\bar{D}$ onto $\bar{D}^{*}$, and $\log p$ is harmonic on $D$.

Proof. Since $u^{*}=v^{*}$, the condition

$$
\mathscr{L}^{2}\left(v^{*-1}(0, M) \cap\left\{y: D v^{*}(y)=0\right\}\right)=0
$$

of Brothers and Ziemer [BZ] is satisfied. By invoking Theorem 1.1 of [BZ], there exists a translation $T: \mathbb{R}^{2} \rightarrow \mathbb{R}^{2}$ such that (a.e.)

$$
u^{*}=v^{*}=v \circ T \text { and } T\left(D^{*}\right)=\mathbb{D}(p) .
$$

Put $F=T^{-1} \circ \widetilde{F}$. Then $F$ is an analytic function on $D$, continuous on $\bar{D}$ with $F(D)=D^{*}$, yielding a conformal representation of $D$ onto $D^{*}$, and $u^{*} \circ F=u$ a.e.

The converse statement follows from the conformal invariance of the Dirichlet integral. 


\section{REFERENCES}

[B] J. Brothers, The isoperimetric theorem, Centre Math. Anal. Preprint R05-87, Australian National Univ., Canberra, 1987.

[BS] H. Behnke and F. Sommer, Theorie der analytischen Funktionen einer komplexen Veränderlichen, Springer-Verlag, New York, Berlin and Heidelberg, 1972.

[BZ] J. Brothers and W. Ziemer, Minimal rearrangements of Sobolev functions, J. Reine Angew. Math. 384 (1988), 153-179.

[C] T. Carleman, Zur Theorie der Minimalflächen, Math. Z. 9 (1921), 154-160.

[EG] L. C. Evans and R. F. Gariepy, Lecture notes on measure theory and fine properties of functions, Kentucky EPSCoR Preprint Series, Univ. of Kentucky, Lexington, Ken., 1987.

[F] H. Federer, Geometric measure theory, Springer-Verlag, New York and Heidelberg, 1969.

[FM] A. Friedman and B. McLeod, Strict inequalities for integrals of decreasingly rearranged functions, Proc. Roy. Soc. Edinburgh 102 (1986), 277-289.

[H] K. Hildén, Symmetrization of functions in Sobolev spaces and the isoperimetric inequality, Manuscripta Math. 18 (1976), 215-235.

[HU] A. Huber, On the isoperimetric inequality on surfaces of variable Gaussian curvature, Ann. of Math. (2) 60 (1954), 237-247.

[K] B. Kawohl, Rearrangements and convexity of level sets in PDE, Lecture Notes in Math., vol. 1150, Springer-Verlag, Berlin-Heidelberg-New York-Tokyo, 1985.

[L] S. Lozinski, On subharmonic functions and their applications to the theory of surfaces. Bull. Acad. Sci. URSS. Ser. Math. [Izv. Akad. Nauk SSSR] 8 (1944), 175-194.

[N] Z. Nehari, On the principal frequency of a membrane, Pacific J. Math. 8 (1958), 285-293.

[S] F. Schulz, Der Faber-Krahnsche Satz für Dirichlet-Gebiete in n Dimensionen, Diplomarbeit, Georg-August-Universität, Göttingen, 1977.

[SP] E. Sperner, Symmetrisierung für Funktionem mehrerer reeller Variablen, Manuscripta Math. 11 (1974), 159-170.

[T] G. Talenti, Best constant in Sobolev inequality, Ann. Mat. Pura Appl. (4) 110 (1976), 353372.

[VS] V. Vera de Serio, Spherical symmetric rearrangements with respect to a nonhomogeneous mass density, Dissertation, The University of Iowa, Iowa City, 1989.

[W] W. Ziemer, Weakly differentiable functions, Springer-Verlag, New York, Berlin and Heidelberg, 1989.

Department of Mathematics, The University of Iowa, Iowa City, Iowa 52242

E-mail address: fschulz@umaxc.weeg.uiowa.edu

Facultad de Ciencias Económicas, U.N.C. Centro Universitario, C. C. 594, 5500 MenDOZA, ARGENTINA 